\title{
SHORT REPORT Molecular epidemiology of Crimean-Congo haemorrhagic fever virus in India
}

P. SINGH, M. CHHABRA*, P. SHARMA, R. JAISWAL, G. SINGH, V. MITTAL, A. RAI AND S. VENKATESH

National Centre for Disease Control, Delhi, India

Received 22 April 2016; Final revision 24 June 2016; Accepted 27 July 2016; first published online 15 August 2016

\section{SUMMARY}

Crimean-Congo haemorrhagic fever (CCHF) is an emerging zoonotic disease in India which is prevalent in neighbouring countries. CCHF virus (CCHFV) is a widespread tick-borne virus which is endemic in Africa, Asia, Eastern Europe and the Middle East. In the present study, samples of clinically suspected human cases from different areas of northern-western India were tested for the presence of CCHFV by RT-PCR through amplification of nucleocapsid (N) gene of CCHFV. Positive samples were sequenced to reveal the prevailing CCHFV genotype(s) and phylogenetic relatedness. A phylogenetic tree revealed the emergence of diverse strains in the study region showing maximum identity with the Pakistan, Afghanistan and Iran strains, which was different from earlier reported Indian strains. Our findings reveal for the first time the emergence of the Asia 1 group in India; while earlier reported CCHFV strains belong to the Asia 2 group.

Key words: Crimean-Congo haemorrhagic fever, $\mathrm{N}$ gene, molecular epidemiology, phylogenetic analysis.

Crimean-Congo haemorrhagic fever (CCHF) is a fatal viral haemorrhagic fever with an overall fatality of 9 $50 \%$ [1]. It is a tick-borne disease caused by the CCHF virus (CCHFV), a member of the genus Nairovirus, family Bunyaviridae. Humans are infected with CCHFV by the bite of infected Ixodid ticks, particularly ticks of the genus Hyalomma or contact with blood or tissue from viraemic livestock or human patients $[2,3]$. The incubation period ranges from 2 to 9 days depending upon the route of exposure and viral inoculum [4]. The spread of CCHF disease may depend upon many factors such as trade and exchange of livestock, transmission via migratory birds

\footnotetext{
* Address for correspondence: Dr M. Chhabra, Division of Zoonosis, National Centre for Disease Control, 22 Sham Nath Marg, Delhi-110054, India.

(Email: malachhabra@yahoo.co.in)
}

infested with infected ticks and subsequent infection of new hosts and transmission in noscocomial settings due to direct contact $[2,3,5,6]$.

The CCHFV genome is triple-segmented having a length of $\sim 19.2 \mathrm{~kb}$. It has negative-sense, singlestranded RNA with segments, i.e. small (S), medium (M), and large (L). The $\mathrm{L}$ segment is $\sim 12 \mathrm{~kb}$ long and encodes for non-structural proteins which comprise the RNA-dependent RNA polymerase gene. The $\mathrm{M}$ (length $\sim 5.7 \mathrm{~kb}$ ) and $\mathrm{S}$ (length $\sim 1.7 \mathrm{~kb}$ ) segments encode for structural proteins for the surface glycoproteins and nucleocapsid, respectively [7, 8]. The CCHFV strains are clustered into seven genetically distinct groups on the basis of the $\mathrm{S}$ segment, i.e. Africa 1 (West Africa, Senegal), Africa 2 (Central, Democratic Republic of the Congo, South Africa), Africa 3 (South and West Africa), Europe 1, Europe 2 (Greece), Asia 1 (Middle East, Pakistan, Iran) and Asia 2 (China, Tajikistan, Kazakhstan) [9-12]. 


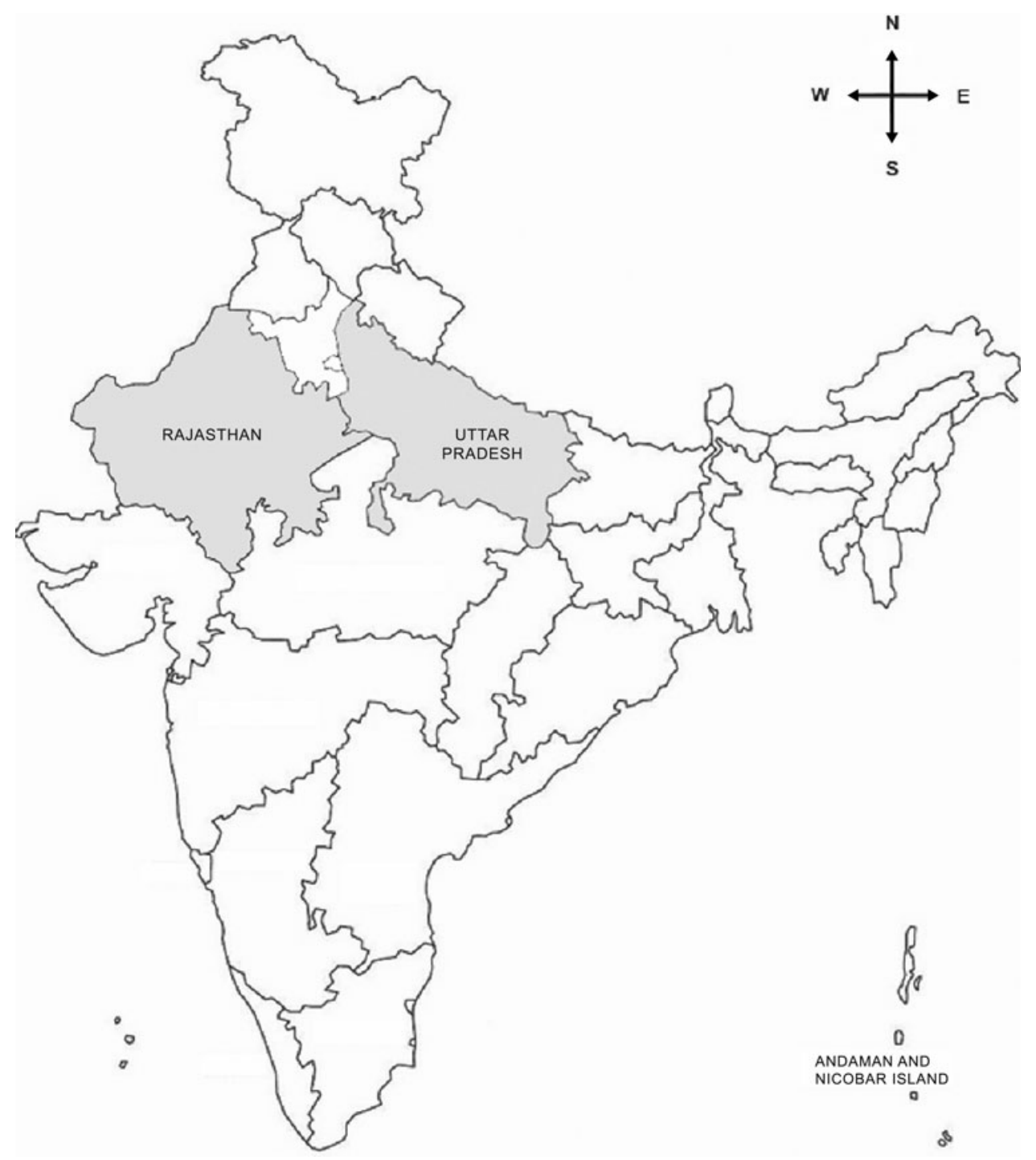

Fig. 1. Map of India. Shaded areas indicate the location of the present study.

Table 1. Details of studied Crimean-Congo haemorrhagic fever virus patients

\begin{tabular}{|c|c|c|c|c|c|c|}
\hline PIDN & Year & Source & Sex & Sign and symptoms & Outcome & Accession no. \\
\hline IND/NCDC/01 & 2015 & Human serum sample & Adult $\mathrm{M}$ & $\begin{array}{l}\text { Fever, arthralgia, } \\
\text { haematemesis, } \\
\text { malena, epistaxis }\end{array}$ & Death & KU041806 \\
\hline IND/NCDC/02 & 2015 & Human serum sample & Adult F & $\begin{array}{l}\text { Fever, epistaxis, } \\
\text { petechia, } \\
\text { splenomegaly }\end{array}$ & Death & KU041807 \\
\hline IND/NCDC/03 & 2015 & Human serum sample & Adult F & Fever, mild illness & Recovered & KU041808 \\
\hline
\end{tabular}

PIND, Patient identification number; M, male, F, female.

The geographical extent of CCHFV is wideranging, which can be correlated by the global distribution of the tick vector. The presence of CCHFV has been reported from many countries of Asia, Africa,
South-East Europe and the Middle East. Frequent outbreaks of CCHF have been reported from countries bordering India such as Pakistan, Afghanistan, and western China. CCHF viral infection was 


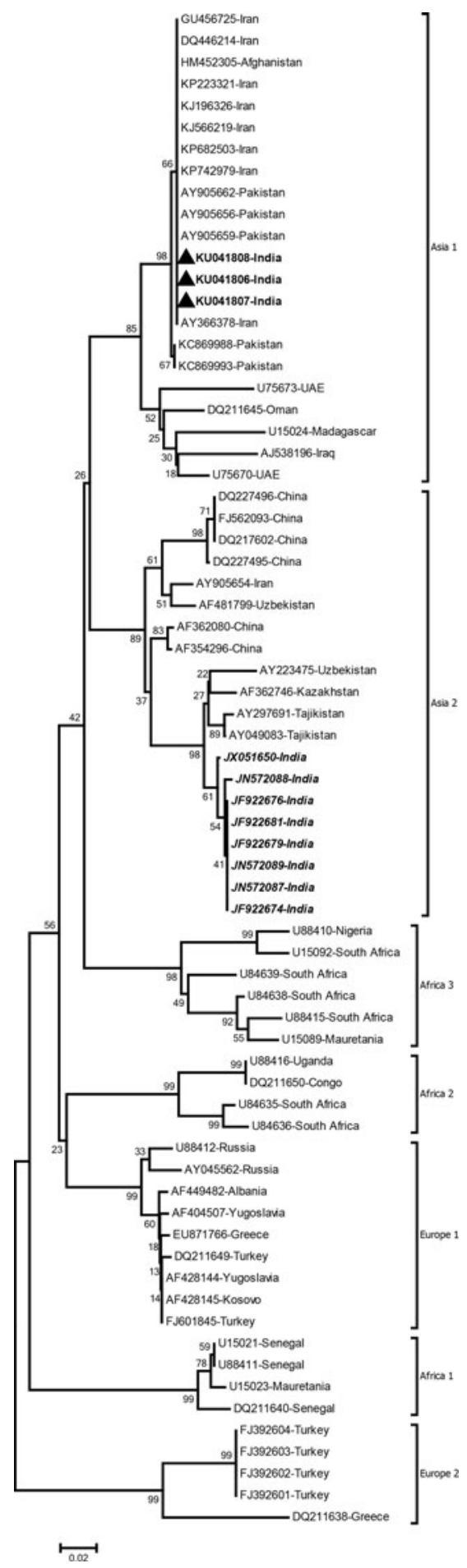

Fig. 2. Phylogenetic tree of Crimean-Congo haemorrhagic fever virus (CCHFV) based on 220-bp nucleotide sequences of the $\mathrm{N}$ gene region generated by the neighbour-joining method. Bootstrap support values (based on 1000 replications) above $50 \%$ are shown at the branch nodes. Naming scheme is accession number followed by name of country. CCHFV strains that were sequenced in the study are shown in bold and indicated by a triangle $(\mathbf{\Delta})$. Bold and italic indicate virus sequences previously reported from India. reported in humans for the first time in Ahmadabad, Gujarat during 2011, although previous seroprevalence studies have shown viral antibodies both in animals and humans [13]. The present study was undertaken for molecular characterization of CCHFV in India using the partial nucleocapsid $(\mathrm{N})$ gene of the S segment.

Serum samples from three clinical cases of haemorrhagic fever, referred to National Centre for Disease Control (NCDC) from different geographical locations in India (Rajasthan and Uttar Pradesh), were tested for CCHFV (Fig. 1). Patients had constitutional symptoms, e.g. fever, arthralgia, and haemorrhagic manifestations, e.g. haematemesis, malena, epistaxis, etc. (Table 1). These samples belonged to focal outbreaks, therefore no ethical clearance was required.

Viral RNA was isolated from the serum samples using QIAamp Viral RNA Mini kit (Qiagen, Germany) according to manufacturer's instructions. The RNA was finally eluted in $50 \mu$ l elution buffer provided in the kit and stored at $-70{ }^{\circ} \mathrm{C}$ until use. Reverse transcription-polymerase chain reaction (RT-PCR) was performed using SuperScript One-step RT-PCR kit (Invitrogen Corp., USA). The outer PCR of $530 \mathrm{bp}$ of the nucleocapsid $(\mathrm{N})$ gene of CCHFV was amplified with forward primer: (CCHF-F2) TGGACA CCT TCA CAA ACT C, and reverse primer: (CCHF-R3) GAC AAA TTC CCT GCA CCA. The nested PCR of 226 bp was performed using forward primer: (CCHF-F3) GAA TGT GCA TGG GTT AGC TC, and reverse primer: (CCHF-R2) GAC ATC ACA ATT TCA CCA GG [14]. Amplified products were visualized on $2 \%$ agarose gel with $0.5 \mu \mathrm{g} / \mathrm{ml}$ ethidium bromide. The PCR products were purified using the QIAquick PCR purification kit according to the manufacturer's instructions (Qiagen). Automated nucleotide sequencing was performed using the Big Dye Terminator Cycle Sequencing ready reaction kit (Applied Biosystems, USA) on an ABI 3130xl automated capillary DNA sequencer (Applied Biosystems). The nested PCR primers, CCHF-F3 and CCHF-R2, were used as forward and reverse primers, respectively, for sequencing. The sequences were aligned, edited and analysed using Clustal W multiple alignment. A BLAST (Basic Local Alignment Search Tool) search was carried out to compare data with already available sequences in the National Centre for Biotechnology Information (NCBI) database. The sequences were submitted to GenBank. Phylogenetic analysis was performed using molecular evolutionary genetics analysis (MEGA) 
software version 6.0 (http://www.megasoftware.net/) and a phylogenetic tree was generated using the neighbourjoining (NJ) method with bootstrap analysis of 1000 replicates using study sequences and other CCHF strains, which were retrieved from GenBank.

In the present study, all three studied serum samples from north-western India were found positive by RTPCR of the $\mathrm{N}$ gene region of CCHFV, which were further sequenced. The sequences were submitted to GenBank and accession numbers were obtained (KU041806-KU041808). The sequences from the study shared $100 \%$ nucleotide identity among themselves. The sequences of the present study shared almost $99-100 \%$ nucleotide identity with strains from neighbouring countries like Pakistan, Afghanistan and Iran. The deduced amino-acid sequences from the study indicated no insertion/deletion in any of the analysed sequences. The study sequences shared a mean $94 \cdot 04 \%$ nucleotide identity with earlier outbreak strains (2010-2011); reported from the western part of India.

The phylogenetic tree revealed that all CCHFVs divide in to seven groups, i.e. Asia 1, Asia 2, Africa 3, Africa 2, Europe 1, Africa 1 and Europe 2 (Fig. 2). The studied CCHFV sequences, clustered with strains previously reported from Pakistan, Afghanistan and Iran from different years, placed in the Asia 1 group. The CCHFV strains from UAE, Oman, Iraq and Madagascar formed a different cluster within the Asia 1 group. However, earlier (2010-11) Indian CCHFV isolates along with a large number of isolates from Tajikistan, Kazakhstan and Uzbekistan formed a separate cluster of the Asia 2 group.

Our findings reveal an emerging diversity of CCHFV strains circulating in India. The current $\mathrm{CCHFV}$ sequences belong to the Asia 1 group and previously reported CCHFV strains belong to the Asia 2 group [13]. This study provides information on gene heterogeneity, phylogenetic relatedness and changing molecular epidemiology of the virus in India. Further studies are necessary to evaluate the impact of the emergence of diverse strains; their clinical manifestation and virulence.

\section{ACKNOWLEDGEMENTS}

The authors are grateful for the financial support received from the National Centre for Disease Control (NCDC) during the course of this study.

\section{DECLARATION OF INTEREST}

None

\section{REFERENCES}

1. Ergonul O. Crimean-Congo hemorrhagic fever. Lancet Infectious Diseases 2006; 6: 203-214.

2. Hoogstraal H. The epidemiology of tick-borne Crimean-Congo hemorrhagic fever in Asia, Europe and Africa. Journal of Medical Entomology 1979; 15: 307-417.

3. Rodriguez LL, et al. Molecular investigation of a multisource outbreak of Crimean-Congo hemorrhagic fever in the United Arab Emirates. American Journal of Tropical Medicine and Hygiene 1997; 57: 512-518.

4. Papa A, et al. Crimean-Congo hemorrhagic fever in Albania, 2001. European Journal of Clinical Microbiology 2002; 21: 603-606.

5. Tonbak S, et al. Crimean-Congo hemorrhagic fever virus: genetic analysis and tick survey in Turkey. Journal of Clinical Microbiology 2006; 44: 4120-4124.

6. Burney MI, et al. Nosocomial outbreak of viral hemorrhagic fever caused by Crimean hemorrhagic fever-Congo virus in Pakistan, January 1976. American Journal of Tropical Medicine and Hygiene 1980; 29: 941-94.

7. Zhou Z, et al. Production of CCHF virus like particle by a baculovirus-insect cell expression system. Virologica Sinica 2011, 26: 338-346.

8. Hewson R, et al. Crimean-Congo haemorrhagic fever virus: sequence analysis of the small RNA segments from a collection of viruses worldwide. Virus Research 2004; 102: 185-189.

9. Carroll SA, et al. Ancient common ancestry of Crimean-Congo hemorrhagic fever virus. Molecular Phylogenetic and Evolution 2010; 55: 1103-1110.

10. Deyde VM, et al. Crimean-Congo hemorrhagic fever virus genomics and global diversity. Journal of Virology 2006; 80: 8834-8842.

11. Anagnostou V, Papa A. Evolution of Crimean-Congo hemorrhagic fever virus. Infection Genetics and Evolution 2009; 9: 948-954.

12. Chamberlain $\mathbf{J}$, et al. Co-evolutionary pattern of variation in small and large RNA segment of Crimean-Congo hemorrhagic fever virus. Journal of General Virology 2005; 86: 3337-3341.

13. Yadav PD, et al. Genetic characterization and molecular clock analyses of the Crimean-congo hemorrhagic fever virus from human and ticks in India, 2010-2011. Infection Genetics and Evolution 2013; 14: 223-231.

14. Drosten C, et al. Molecular diagnostics for viral hemorrhagic fevers. Antiviral Research 2003; 57: 61-87. 